\title{
Unknown Risk Factor
}

National Cancer Institute

\section{Source}

National Cancer Institute. Unknown Risk Factor. NCI Thesaurus. Code C159865.

The risk factor status is unknown. 